\title{
Analysis on linac quadrupole misalignment in FACET commissioning 2012
}

\author{
Yipeng Sun \\ SLAC National Accelerator Laboratory, Menlo Park, California 94025, USA
}

July 5,2012

\begin{abstract}
In this note, the analysis on linac quadrupole misalignment is presented for the FACET linac section LI05-09 plus LI11-19. The effectiveness of the beam-based alignment technique is preliminarily confirmed by the measurement.
\end{abstract}

\section{Overview}

Beam-based alignment technique was adopted at SLAC linac since SLC time [1]. Here the beambased alignment algorithms are further developed and applied in the FACET commissioning during 2012 run.

\section{FACET optics and MATLAB simulation results}

FACET is a facility dedicated for beam-driven plasma wake field acceleration experiment and other programs, which uses the first two kilometers of SLAC linac [2]. The TWISS beta function in FACET linac LI05-19 is shown in Figure 1 (left), and the beam energy (max. of $22 \mathrm{GeV}$ ) is shown in Figure 1 (right).

The details of the beam-based alignment algorithms are discussed in reference [3] and reference [4], which are not repeated here. In principle, it employs first order transport matrix to solve beam's position and angle, as shown in formulae 1 and formulae 2.

$$
\begin{gathered}
x_{i}=R_{12_{i, 1}} \theta_{1}-\sum_{j=1}^{i-1} R_{12_{i, j}} K_{j} x_{q, j} \\
x_{i}{ }^{\prime}=R_{22_{i, 1}} \theta_{1}-\sum_{j=1}^{i-1} R_{22_{i, j}} K_{j} x_{q, j}-x_{q, i} K_{i} / 2
\end{gathered}
$$

The whole linear system is then expressed in matrix format, as shown in formulae 3 . 


$$
\mathbf{R} \cdot \mathrm{Q}=\mathrm{B}
$$

The matrix $\mathbf{R}, \mathbf{Q}$ and $\mathbf{B}$ denote

- $\mathbf{R}$ combined $\mathrm{R}$ matrix, $n \times n$ sparse matrix

- $\mathrm{Q}$ quad offsets+initial beam offset, $n \times 1$

- B BPM reading, $n \times 1$

- B includes BPM to Q offset error, and BPM measurement error

This linear system can be solved by Row reduction (Gaussian elimination).

The schematic of this system is shown in Figure 2. The simulation program written in MATLAB is first benchmarked with ELEGANT simulation, where good agreement is achieved as shown in Figure 3. It is noted that outstanding quad offset of $2 \mathrm{~mm}$ can be effectively detected, given 200 $\mu \mathrm{m}$ BPM to quad offset and $300 \mu \mathrm{m}$ normal quad offset, as shown in Figure 4.

\section{Analysis on FACET linac data LI05-09 plus LI11-19}

The MAD8 model used in constructing the matrix $\mathbf{R}$ is first benchmarked with the FACET machine by comparing oscillation data (launched by two dipole correctors in horizontal and vertical plane respectively).

Then the linac is divided into sections LI05-06, LI07-09, LI11-13, LI14-16 and LI17-19, and the quad offset is calculated in each section. This technique is to minimize the impact from optics errors which may sum up.

For each section, three sets of data are taken on different dates. One set of data includes the BPM reading, the steering dipole corrector strength and the quadrupole strength.

The MATLAB algorithm has functions: (Given BPM measurement data and dipole corrector settings from any steering method )

- read in MAD optics model and BPM data

- subtract dipole corrector contribution in BPM data; get new BPM data (only from quads kicks and initial beam angle)

- calculate each quad offset and initial beam angle (N-1 BPMs $\rightarrow$ N-2 quads + initial beam angle)

In Figure 5 three sets of data (BPM readings X/Y, and steering dipole corrector strength $\mathrm{X} / \mathrm{Y}$ ) are shown for LI05-06. It is observed that they are quite different.

Then following the steps discussed above, the quad misalignment is calculated (Reference line: QUAD:LI05:201 to QUAD:LI06:901 ). From the results shown in Figure 6, it is observed that the convergence of this algorithm is good.

These quad offsets data (amended with BPM-quad offset measurement, by 'bow-tie' or another new technique [5]) are given to alignment crews who then move the quad with large offsets accordingly. Positive moves are up (y, vertical plane) and to the wall (x, horizontal plane). 
After the move, the quad misalignment is measured and calculated again. The difference of the two sets of calculated quad offset from measurement agrees well with the amount that the quads are moved, as listed in Table 1.

Table 1: Quad movement agrees well with measurement.

\begin{tabular}{l|c|c|c|c}
\hline & X moved [mil] & $\Delta X$ measured [mil] & Y moved [mil] & $\Delta$ Y measured[mil] \\
\hline Quad Li05 601 & 10 & 13 & 20 & 3 \\
Quad Li05 701 & - & -5 & 25 & 30 \\
Quad Li05 801 & -30 & -24 & - & -4 \\
Quad Li05 901 & -15 & -4 & -30 & -49 \\
Quad Li06 301 & - & 0 & -25 & 26 \\
Quad Li06 501 & - & -1 & -33 \\
\hline
\end{tabular}

Possible error sources are:

- quad strength error (systematic and random error)

- dipole corrector strength scaling error

- BPM random error (resolution, roughly $50 \mu \mathrm{m}$ for SLAC linac)

- BPM reading scaling error (systematic error)

- Effects not included in the algorithm, wake fields, RF kicks etc.

- error in moving the quad by alignment crews

The quad misalignments calculated for FACET linac LI07-09, LI11-13, LI14-16 and LI17-19 are shown in Figure 7 to Figure 10 below.

The calculated quad misalignment in LI17-19 is very suspicious which needs further analysis. It may be due to a wrongly selected reference axis, as shown in Figure 11.

\section{Acknowledgement}

The author would like to thank C. Adolphsen, F.-J. Decker, A. Latina, N. Lipkowitz, Y. Nosochkov, U. Wienands, M. Woodley, G. Yocky for helpful discussions. Special thanks go to C. Adolphsen for his guidance and support.

This work was supported by the DOE under Contract DE-AC02-76SF00515.

\section{References}

[1] Chris Adolphsen et al., SLAC-PUB-4902 (1989); SLAC-PUB-5439 (1991); SLAC-PUB-6233 (1993).

[2] C. Joshi et al., 'Plasma wakefield acceleration experiments at FACET,' New Journal of Physics 12, 055030 (2010).

[3] Yipeng Sun, SLAC-PUB-14466 (2012). 

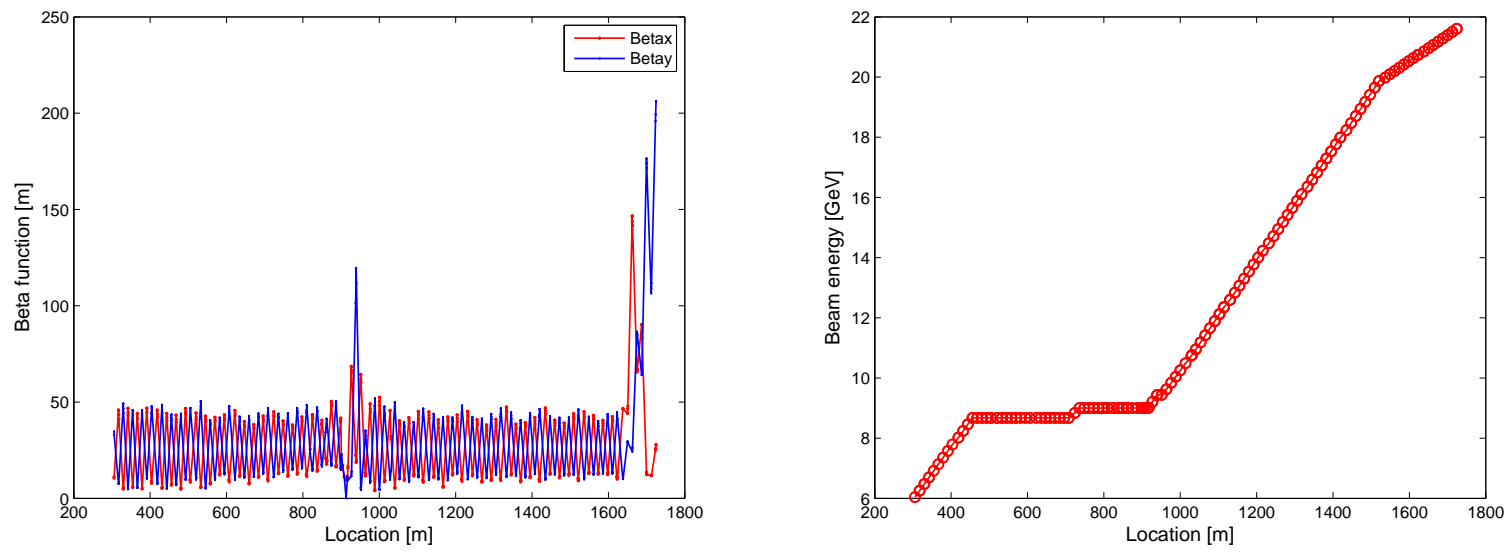

Figure 1: FACET linac LI05-19. Left: TWISS beta function; Right: beam energy (acceleration) along the linac.

[4] Yipeng Sun, SLAC-PUB-14471 (2012).

[5] Franz-Josef Decker, private communication (2012). 

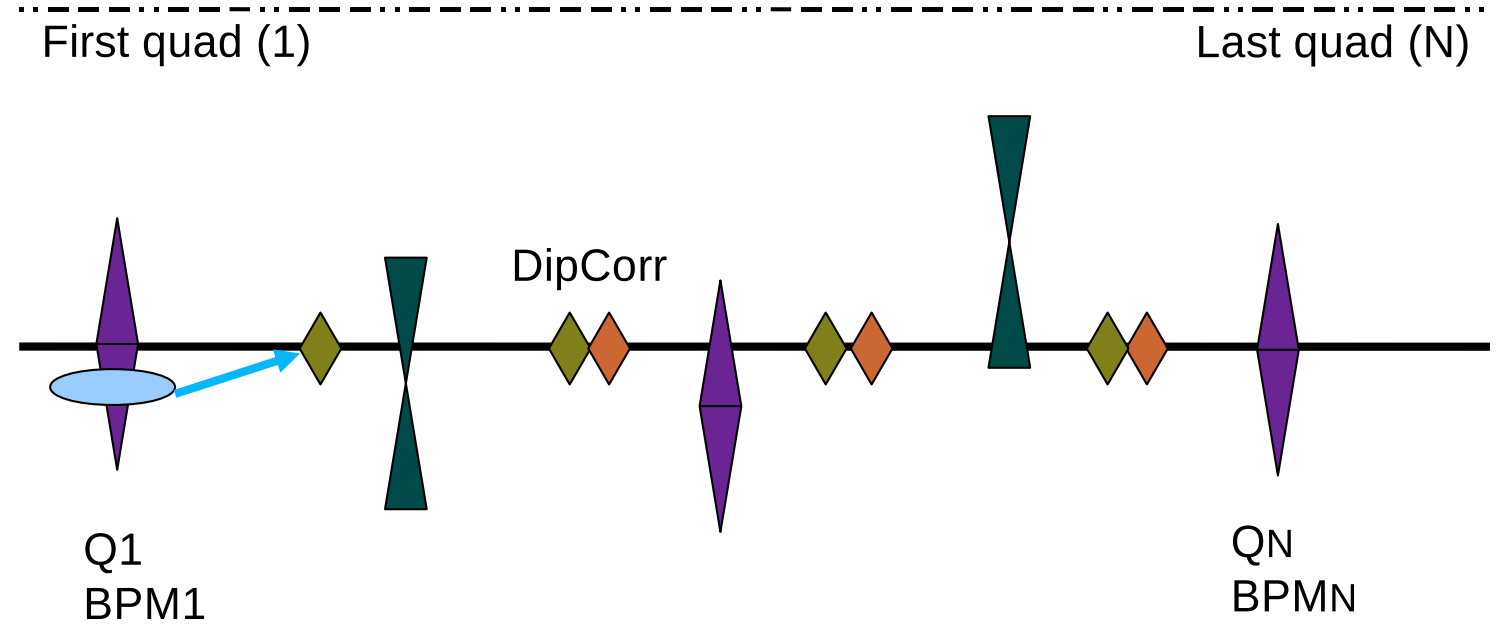

Figure 2: Schematic of the linac system, with quadrupoles, BPMs, steering dipole correctors and drift spaces. Reference axis from the center of first quad to last quad.
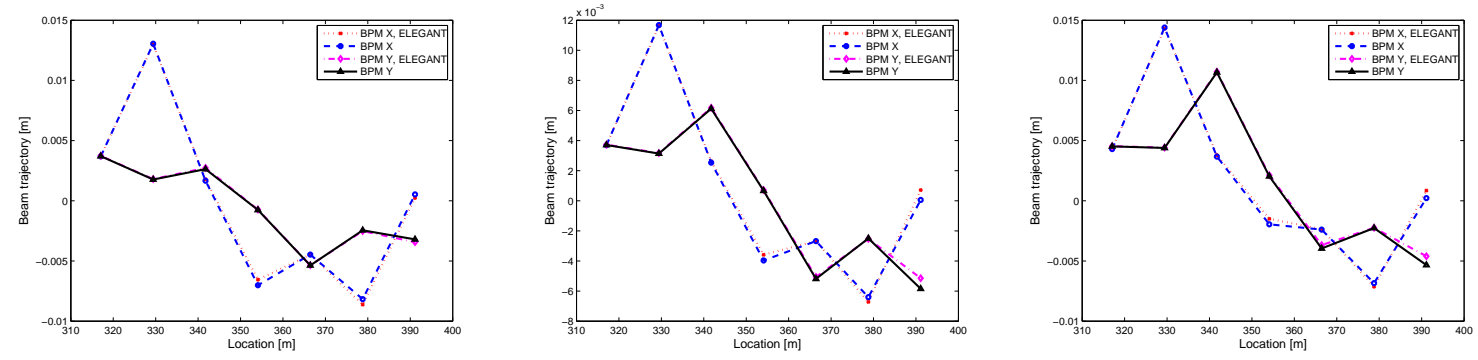

Figure 3: Benchmark of the MATLAB script with ELEGANT simulation results show good agreement. Small difference is due to thin-lens (MATLAB script) and thick-lens (ELEGANT) adopted for magnets model. Left: with initial beam angle $300 \mu \mathrm{m}$; middle: with initial beam angle $300 \mu \mathrm{m}$, and RMS quad offset of $900 \mu \mathrm{m}$; right: with initial beam angle $300 \mu \mathrm{m}$, RMS quad offset of 900 $\mu \mathrm{m}$ and RMS dipole corrector strength of $50 \mu \mathrm{rad}$. 


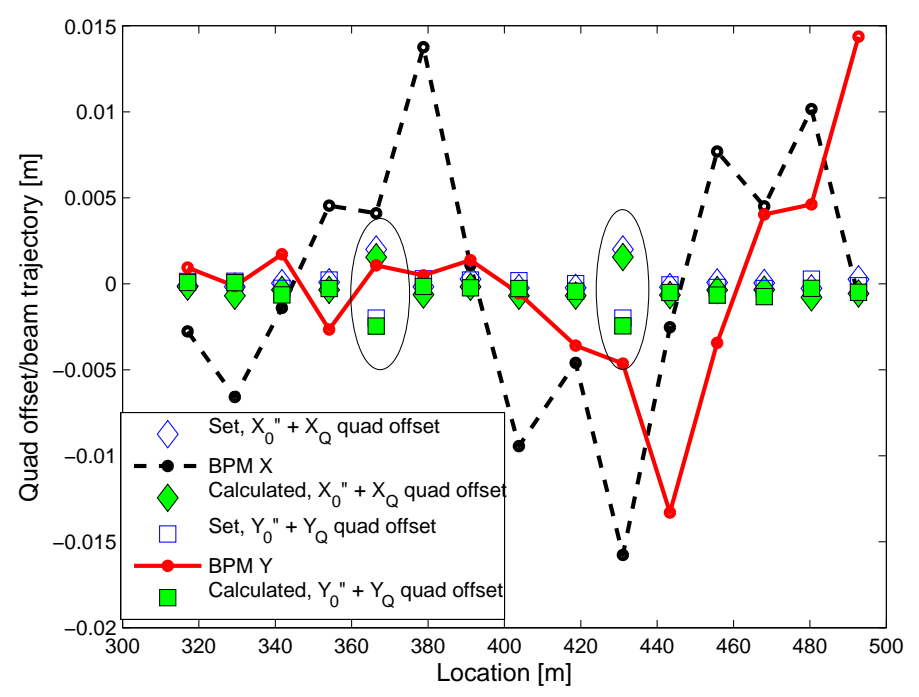

Figure 4: $200 \mu \mathrm{m}$ is assumed for the offset between BPM (Beam Position Monitor) electrical center and quadrupole magnetic center, and $300 \mu \mathrm{m}$ for quad offset (RMS). The simulation shows that outstanding quad offset of $2 \mathrm{~mm}$ can still be detected, as crossed by the eclipse.
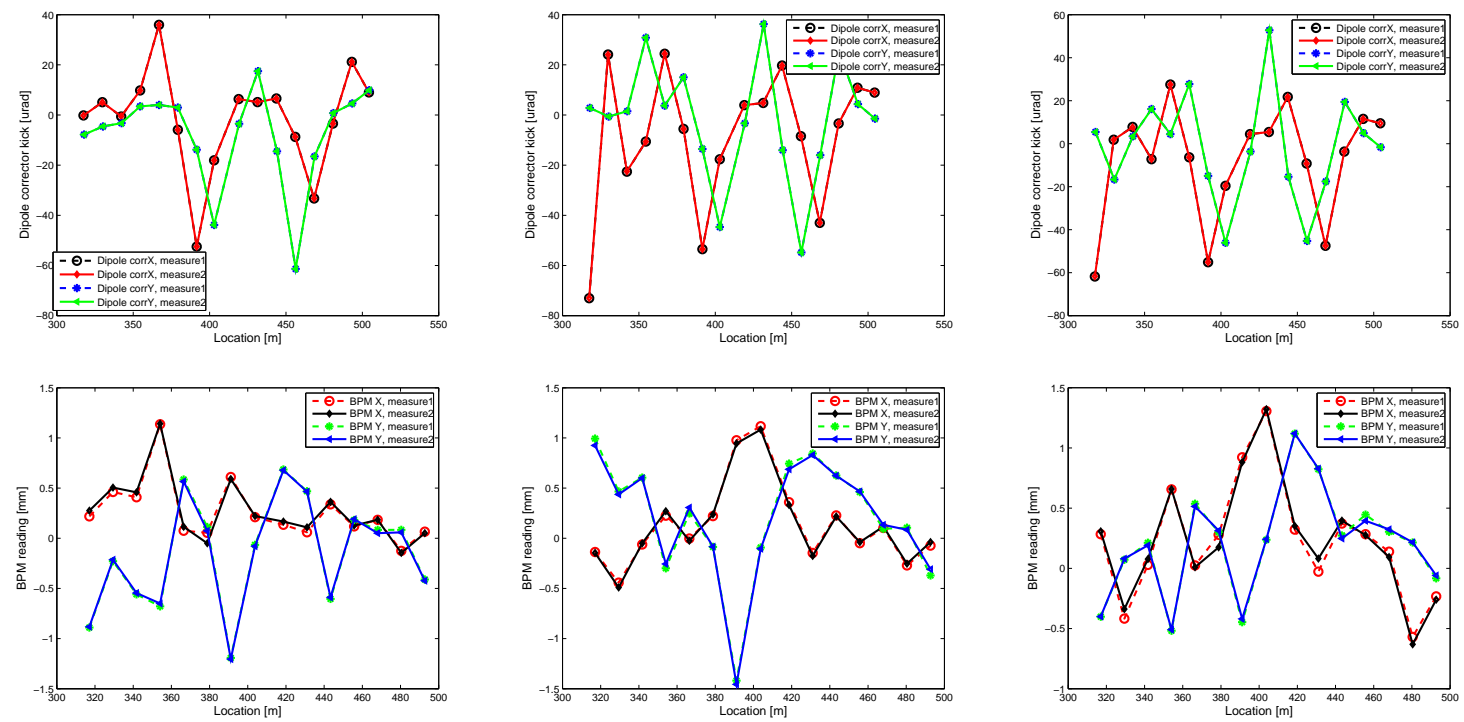

Figure 5: BPM readings X/Y, and steering dipole corrector strength X/Y of LI05-06. Left: April 02, 2012; middle: April 12, 2012; right: April 19, 2012. 

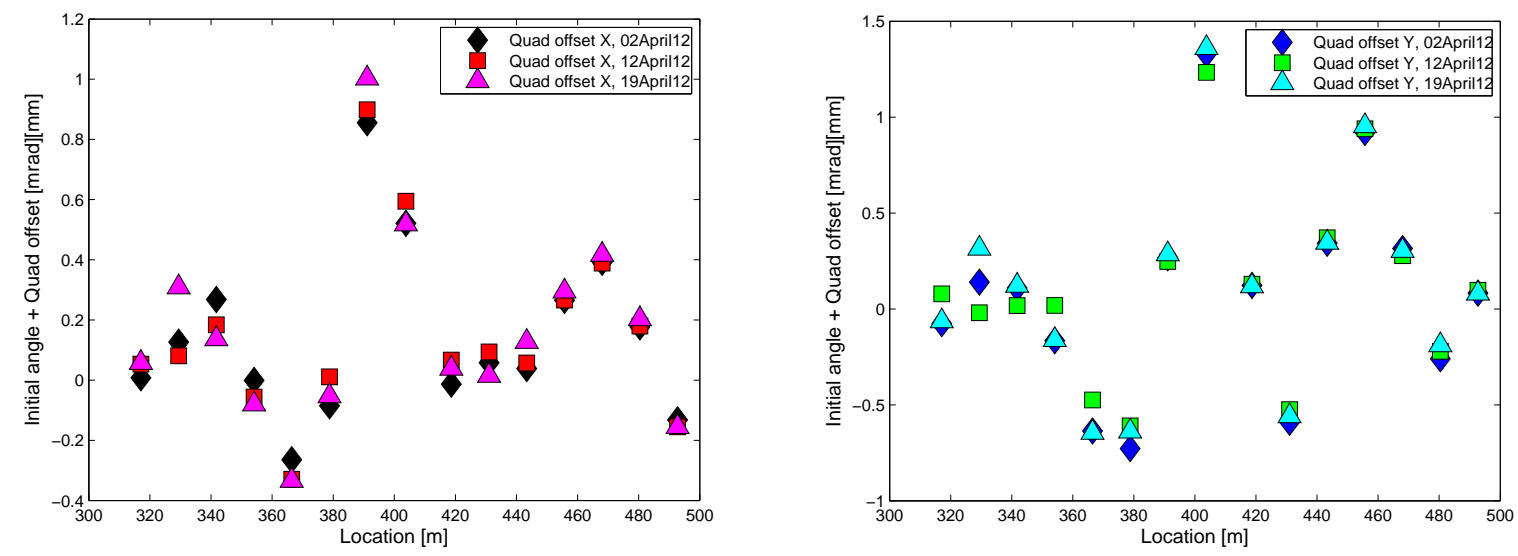

Figure 6: Calculated quad misalignment in LI05-06. Reference axis: center of first quad to last quad.
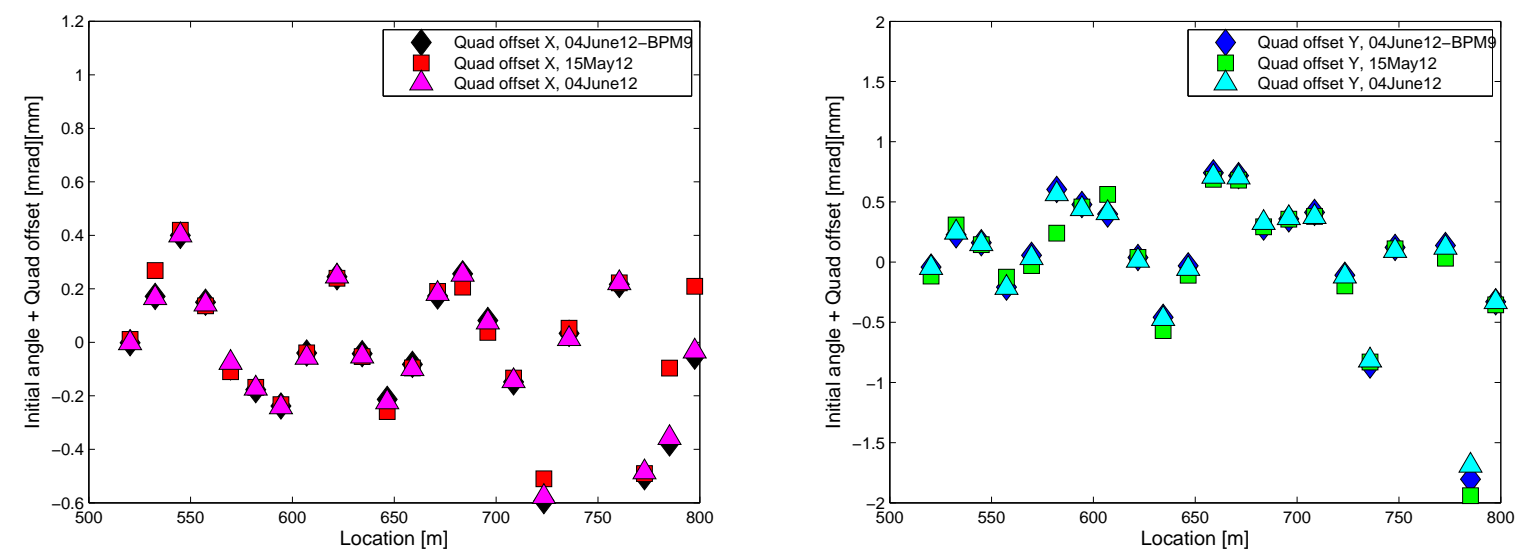

Figure 7: Calculated quad misalignment in LI07-09. Reference axis: center of first quad to last quad. 

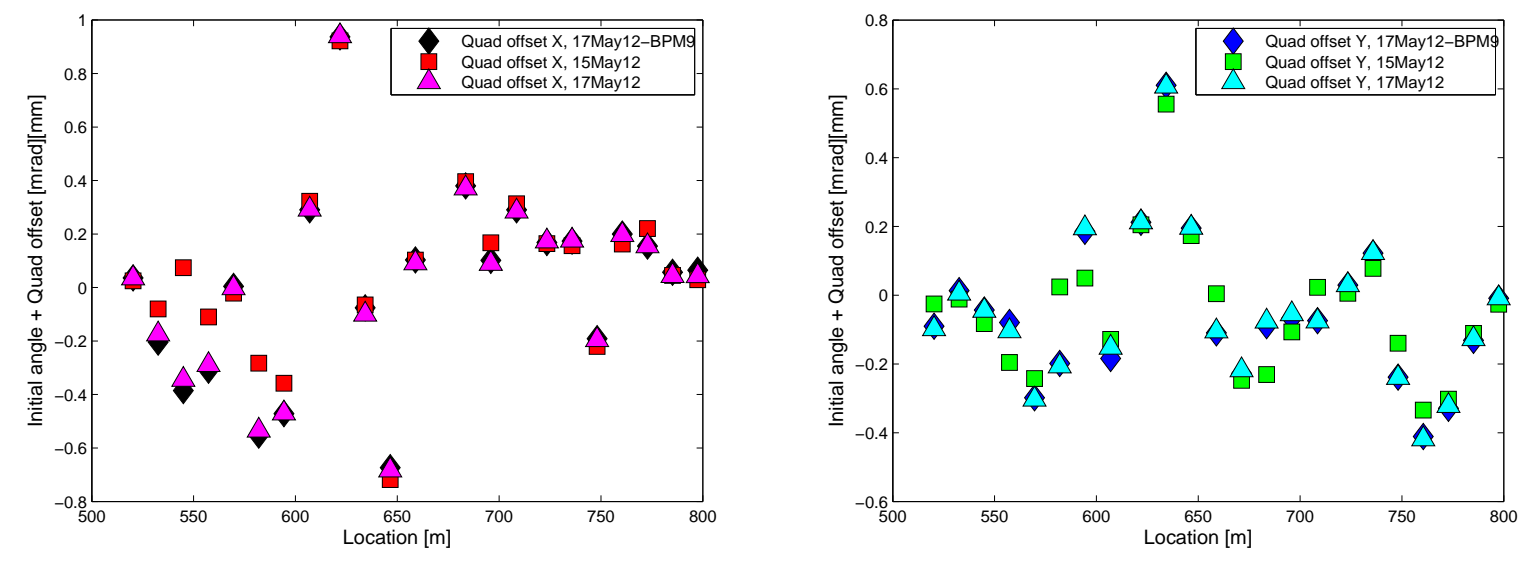

Figure 8: Calculated quad misalignment in LI11-13. Reference axis: center of first quad to last quad.
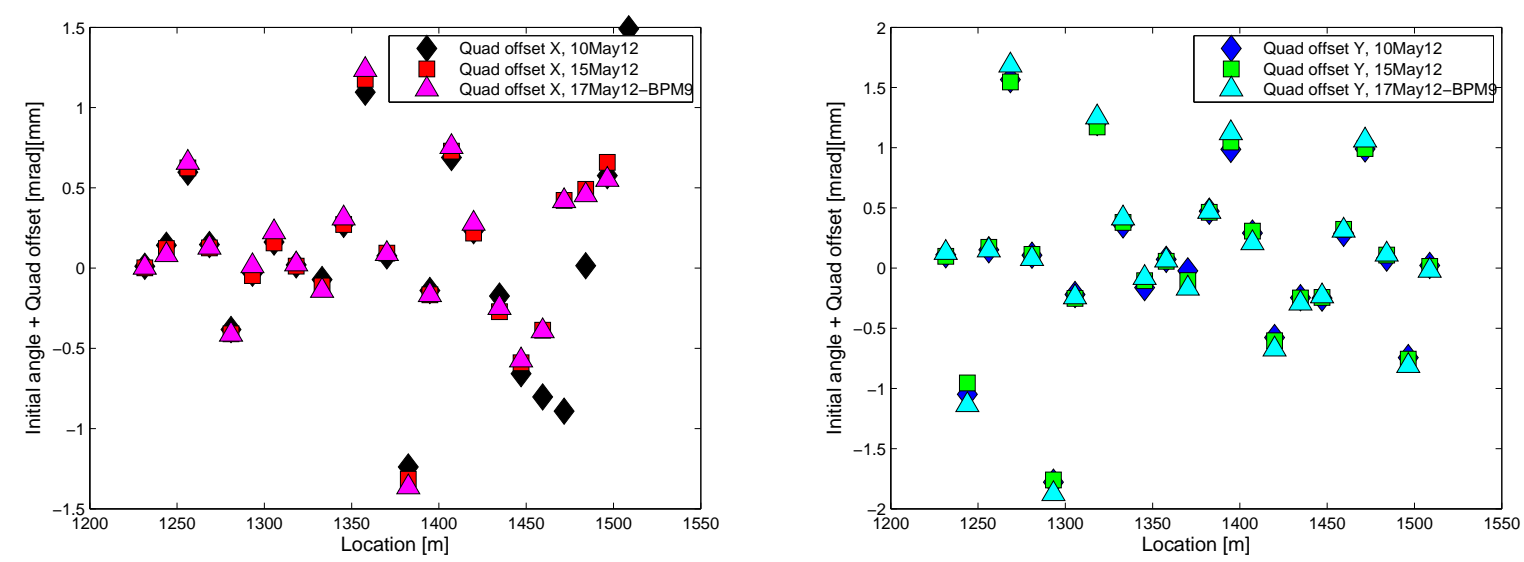

Figure 9: Calculated quad misalignment in LI14-16. Reference axis: center of first quad to last quad. 

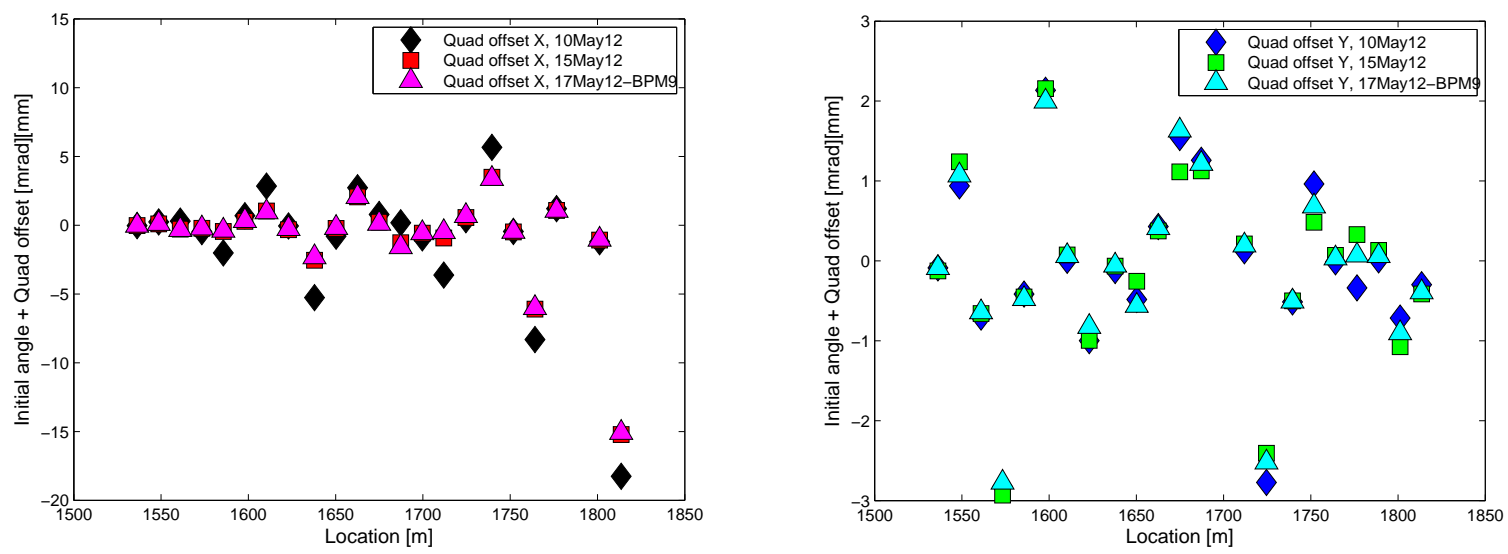

Figure 10: Calculated quad misalignment in LI17-19. Reference axis: center of first quad to last quad.

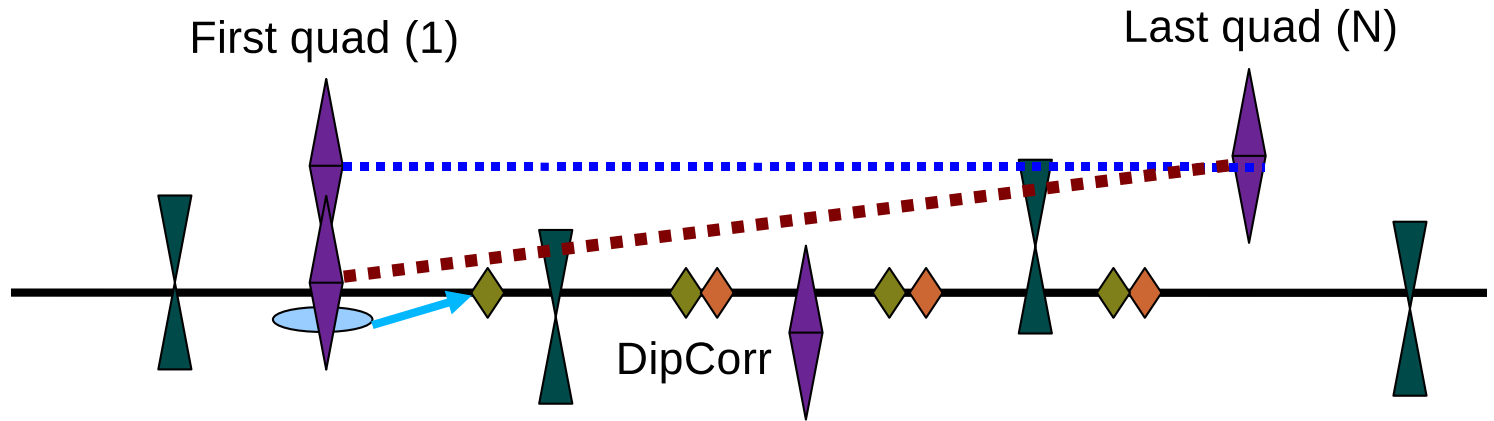

Figure 11: Schematic of the linac system for two cases of wrongly selected reference axis (blue and red curve), with quadrupoles, BPMs, steering dipole correctors and drift spaces. Reference axis from the center of first quad to last quad. 